\title{
Diameter and charge of the first droplet emitted in electrospray
}

M. Rubio, ${ }^{1}$ S. H. Sadek, ${ }^{1}$ A. M. Gañán-Calvo, ${ }^{2}$ and J. M. Montanero ${ }^{1}$

1) Departmento de Ingeniería Mecánica, Energética y de los Materiales and

Instituto de Computación Científica Avanzada (ICCAEx),

Universidad de Extremadura, Avda. de Elvas s/n, E-06071 Badajoz, Spain

${ }^{2)}$ Departamento de Mecánica de Fluidos e Ingeniería Aeroespacial,

Universidad de Sevilla, E-41092 Sevilla, Spain

(Dated: 25 January 2021)

The first droplet produced by a low-conductivity pendant/sessile droplet subject to a strong electric field is particularly important at the fundamental level because, in contrast to steady electrospray phenomena, its ejection entails complex charge relaxation and electrokinetic processes. Besides, it is technologically relevant because of its very small diameter and large electric charge per unit volume. In this work, we present an experimental technique to measure with unprecedented accuracy the diameter of the droplet, and to determine for the first time its electric charge. We discuss both the advantages of our technique over possible alternatives and the limitations of the method. The proposed method is applied to two alcohols with electrical conductivities of the order of a few $\mu \mathrm{S} / \mathrm{m}$. The high sensitivity of our experimental technique allows us to determine the influence of both the magnitude and polarity of the applied voltage on the size and charge of the ejected droplet. The electric charge of the first-emitted droplet lies in the interval $0.51 \lesssim q / q_{R} \lesssim 0.66\left(q_{R}\right.$ is the Rayleigh limit of charge) for the two liquids analyzed. These experimental values are slightly larger than those obtained from theoretical predictions. The value of $q / q_{R}$ for the first droplet is very relevant because it can be regarded as an upper bound of those of the droplets subsequently emitted in the cone-jet mode of electrospray.

\section{INTRODUCTION}

Rayleigh ${ }^{1}$ claimed in his pioneering work that a liquid droplet with an electric charge larger than $q_{R}=\left(8 \pi^{2} \varepsilon_{o} \gamma d^{3}\right)^{1 / 2}$ ( $\gamma$ is the surface tension, $\varepsilon_{o}$ is the vacuum electrical permittivity, and $d$ is the droplet diameter) destabilizes due to the growth of the quadrupole oscillation mode because the repulsive Coulomb force exceeds the attractive surface tension force (the so-called Rayleigh limit). He also predicted that higher multipole oscillations can cause the ejection of very thin jets, known as Rayleigh jets. Taylor ${ }^{2}$ described experimentally the ejection of Rayleigh jets from the conical point of electrified films, which constituted the first observation of electrohydrodynamic tip streaming ${ }^{3}$. Since then, the emission of Rayleigh jets has been continuously examined in various experimental configurations, such as levitated bubbles and droplets ${ }^{5-8}$, electrified films ${ }^{9}$, and sessile ${ }^{10}$ and pendan droplets ${ }^{11-14}$

The unsteady disintegration of charged and neutral drops under externally applied electric fields constitutes a fundamental electrohydrodynamic problem with enormous relevance in natural and technological processes. This tip streaming phenomenon has been analyzed theoretically over the last decades ${ }^{13-21}$ assuming perfect volumetric charge relaxation and ohmic conduction (the so-called leaky-dielectric model $)^{13,16,17,21}$. Some studies have also considered certain electrokinetic effects along the process, including volumetric charge relaxation phenomena ${ }^{14,18-20}$.

The first tiny droplet produced by the disintegration of electrified drops (Fig. 1) is particularly important at both fundamental and technological levels because of its very small diameter and large electric charge per unit volume. During the ultra-fast ejection of that droplet, a fresh free surface is created from the stretching of the mother drop apex with a characteristic time comparable to the electric relaxation time ${ }^{14}$. The Debye layer formed at the free surface over the first stage of

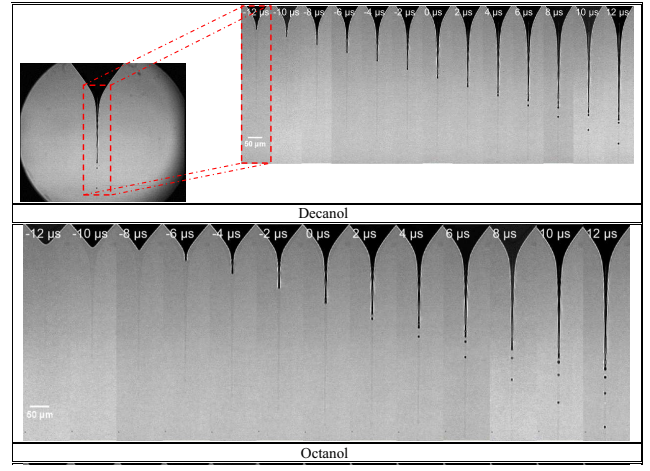

FIG. 1. Images of the first droplet ejected.

the process is suddenly disrupted, and so is the ohmic injection of charge into the newly created free surface. This charge injection plays a crucial role to sustain the droplet formation. Therefore, the failure of the ohmic conduction is expected to leave its mark on the size and charge of the emitted droplets. For this reason, the phenomenon described above constitutes an ideal candidate to challenge the current electrohydrodynamic models.

As a first approximation, the ejection of the first droplet is assumed to be a local process, which takes place in a region much smaller than the parent drop. In this case, one can assume that the geometrical variables do not significantly affect the phenomenon ${ }^{17}$. The applied electric field magnitude is expected to have little or at most moderate influence because it must lie within a relatively narrow range for the ejection to occur. Under these approximations, the parameters which essen- 
tially characterize the problem are the liquid properties (density $\rho$, viscosity $\mu$, surface tension $\gamma$, electrical conductivity $K$, and electrical permittivity $\varepsilon_{i}$ ) and the electrical permittivity $\varepsilon_{o}$ of the dielectric environment (vacuum or gas). The characteristic length $d_{o}=\left(\gamma \varepsilon_{o}^{2} / \rho K^{2}\right)^{1 / 3}$, time $t_{o}=\varepsilon_{o} / K$, velocity $v_{o}=\left(\gamma K / \rho \varepsilon_{o}\right)^{1 / 3}$, electric field magnitude $E_{o}=\left(\gamma / d_{o} \varepsilon_{o}\right)^{1 / 2}$, and the electric charge $q_{o}=\varepsilon_{o} E_{o} d_{o}^{2}$ are defined from those physical properties. These characteristic scales can be derived from the so-called physical symmetries of the Taylorcone jets ${ }^{22}$, which involve the conservation of mass, axial momentum, energy, and the balance between surface convection and bulk condition of charge in the cone tip. The problem is governed by the electrohydrodynamic Reynolds number $\delta_{\mu}=\rho d_{o} v_{o} / \mu$ and relative permittivity $\varepsilon=\varepsilon_{i} / \varepsilon_{o}^{23}$. The dimensionless diameter $d / d_{o}$ and electric charge ratio $q / q_{R}$ of the first-ejected droplet are expected to depend on those two numbers exclusively.

Collins et al. ${ }^{17}$ analyzed the problem described above by numerically solving the leaky-dielectric model, and concluded that $q / q_{R}=0.44$. Gañan-Calvo et al. ${ }^{14}$ rationalized their numerical and experimental results in terms of the selfsimilar collapse of the drop's tip, the lack of full charge relaxation from the bulk to the free surface, and the balance between inertia, axial viscous stresses, surface tension, and electrostatic suction during the jet's evolution. This analysis led to the scaling laws $d / d_{o}=0.6 \zeta_{d}$ and $q / q_{R}=0.48 \zeta_{R}$, where $\zeta_{d}=\delta_{\mu}^{-1 / 3} \varepsilon^{5 / 12}$ and $\zeta_{R}=\delta_{\mu}^{-1 / 6} \varepsilon^{-1 / 24}$.

The validity of the two approximations described above can be tested by looking at the size and charge of the first-emitted droplet for different applied voltages and when the polarity of the applied electric field is inverted. The magnitude of the applied voltage determines the dynamics of the non-local evolution of the parent drop deformation, establishing the ultimate boundary conditions for the local ejection. Thus, although the appearance of local ejection is rather restrictive for the applied voltage, the influence of this quantity may be noticeable through the non-local deformation mode prior to emission. In addition, the different mobilities of the ionic species in the liquid bulk may lead to different electrokinetic response times where the charge transport is most compromised (i.e. where velocities and accelerations become larger). Specifically, the rate at which the charge migrates to the free surface during the first droplet formation may significantly depend on the applied polarity, which dictates the direction of the electroosmotic drift. Therefore, the polarity of the applied voltage should affect the outcome of the process.

Neither the voltage nor the polarity effects mentioned above are contemplated in the leaky-dielectric model commonly used to describe this phenomenon ${ }^{17}$ or in the approximation described by Gañán-Calvo et al. ${ }^{14}$. In fact, these two approaches make use of the equilibrium (polarity-independent) liquid conductivity to calculate the transfer of charge from the bulk to the free surface. Since the voltage and polarity effects are expected to be relatively small, both the diameter and electric charge of the first-emitted droplet must be measured with a small uncertainty to unveil those effects, which constitutes a considerable experimental challenge.

Despite its relevance at both the fundamental and techno- logical levels, the electric charge of the first droplet that precedes the steady ejection of the electrospray cone-jet mode has not as yet been determined experimentally. In this work, we will develop an experimental method to measure the charge of the first droplet emitted when a low-conductivity pendant droplet is subject to an externally applied electric field (Fig. 1). Also, we will measure the diameter and velocity of the droplet with an unprecedented accuracy, which will allow us to examine the influence of the magnitude and polarity of the applied electric field, factors commonly neglected in this problem.

\section{EXPERIMENTAL METHOD}

\section{A. Experimental setup}

Figure 2 shows the experimental setup used in this work. We placed horizontally three plates made of stainless steel. The upper and lower plates were $3.6 \mathrm{~cm}$ in diameter and 3.31 $\mathrm{mm}$ in thickness. The middle plate had a quadrangular shape of $3.6 \mathrm{~cm}$ in side and $0.33 \mathrm{~mm}$ in thickness. This plate was introduced to screen the electric field perturbation caused by stretching pendant droplet. In this way, the electric field between the middle and lower plates is practically uniform. A copper disk of radius $R_{0}=2 \mathrm{~mm}$ and thickness $0.7 \mathrm{~mm}$ was co-axially attached to the upper plate. This element was introduced to ensure the anchorage of the triple contact line. Both the upper plate and the disk had circular orifices of diameter $200 \mu \mathrm{m}$ at their centers. The middle plate was separated by a distance of $4.31 \mathrm{~mm}$ from the upper one and had an orifice $200 \mu \mathrm{m}$ in diameter at its center too. The distance between the middle and lower plate was $1.77 \mathrm{~mm}$. The distances between the electrodes must be large enough to avoid the electric arc between them, and small enough to produce a sufficiently intense electric field. We connected the three plates with two resistors whose electrical resistances were selected to establish an electric field of approximately the same magnitude in the two gaps between the plates. These resistances were sufficiently high for the electric current to be smaller than the maximum value supplied by the voltage amplifier, and low enough to ensure that the circuit resistance-capacitance time was much smaller than any electrohydrodynamic time of the problem. The lower plate was grounded.

A DC voltage drop was set between the upper and lower (grounded) plates using a voltage amplifier (Trek, Model 5/80). Positive/negative polarity corresponds to an electric field pointing in the same/opposite direction as/to the liquid ejection. A function generator (Keysight, Model 33210A 10 $\mathrm{MHz}$ ) was used to trigger both the voltage amplifier and the high-speed camera described below. The pendant droplet was formed by injecting liquid with a syringe pump (KD Scientific, Legato 210 Series) through the small orifice located in the center of the upper electrode.

Digital images of pendant drop tip and the emitted droplet were taken using an ultra-high-speed video camera (KIRANA$5 \mathrm{M})$ equipped with optical lenses (12X NAVITAR) and a microscope objective (10 X MITUTOYO). The images consisted 


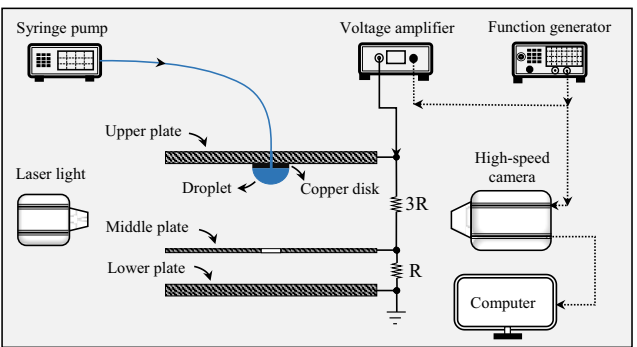

FIG. 2. Experimental setup used in this work.

of $924 \times 768$ pixels and were acquired at either $5 \times 10^{5}$ or $5 \times 10^{6}$ fps depending on the case. The camera could be displaced both horizontally and vertically using a triaxial translation stage with one of its horizontal axes (axis $x$ ) motorized (THORLABS Z825B) and controlled by the computer, which allowed as to set the droplet-to-camera distance with an error smaller than $200 \mathrm{~nm}$. The camera was illuminated with a laser (SI-LUX 640, SPECiALISED IMAGING) synchronized with the camera, which reduced the effective exposure time down to $100 \mathrm{~ns}$. Different optical magnifications were required to measure the emitted droplet diameter and charge. For this reason, those two quantities could not be measured simultaneously in the same experimental realization. We conducted two sets of experiments: one for measuring the droplet diameter, and another to determine the electric charge. The respective magnifications were $70 \times$ and $14 \times$, which resulted in 0.44 and $2.17 \mu \mathrm{m} /$ pixel, respectively. All the elements of the experimental setup were mounted on an optical table with a pneumatic anti-vibration isolation system to damp the vibrations coming from the building.

Table I shows the properties of the working liquids and the volume $\mathscr{V}$ of the pendant droplet. The density $\rho$, viscosity $\mu$, and relative electrical permittivity $\varepsilon$ were taken from the literature. The surface tension was measured with the TIFA (Theoretical Image Fitting Analysis) method ${ }^{24}$, while the electrical conductivity was determined by applying a voltage difference between the ends of a borosilicate capillary filled with the working liquid. We conducted experiments for two values of the voltage drop $V_{0}$ between the upper and middle plates: the minimum voltage $V_{0}=V_{0 \min }$ for which the phenomenon was observed, and the maximum value $V_{0}=V_{0 \max }$ supplied by the voltage amplifier. Table I shows the voltage values for those experiments in which the first droplet diameter could be determined. Due to its relatively large conductivity, the pendant drop of propanol emitted a tiny droplet in the range of voltages analyzed. The diameter of that droplet could not be determined with our image acquisition system. Table I also shows the values of the pendant droplet volume $\mathscr{V}$ in each experiment. This value was slightly reduced for $V_{0}=V_{0 \max }$ to make the first droplet detach from the ejected thread before crossing the middle plate.

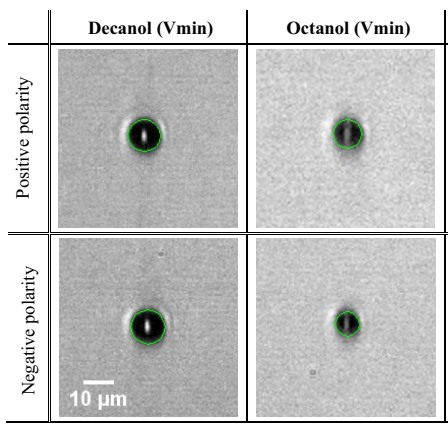

FIG. 3. Images of the first droplet ejected with the two polarities. The green circle approximately corresponds to the circle fitted to the droplet shape.

\section{B. Experimental procedure}

As mentioned above, we conducted two sets of experiments to measure the droplet diameter and charge independently. The droplet was ejected following the same procedure in both cases. The pendant droplet was inflated until the prescribed volume was reached (see Table I). We checked that the volume was practically the same in all the repetitions of the experiment by looking at the position of the droplet apex. Then, a constant voltage drop was applied until the first ejected droplet reached the lower electrode. The camera was synchronized with the power supply to record images at a certain instant after the voltage drop was applied. The camera delay was selected in the interval 8-19 ms depending on the liquid and on whether either the droplet diameter or charge was to be determined.

Due to its small size, surface tension makes the droplet adopt a quasi-spherical shape despite the action of the aerodynamic and electrical forces. In fact, the Weber number $\mathrm{We}=\rho_{a} v_{d}^{2} d / \gamma\left(\rho_{a}\right.$ is the air density and $v_{d}$ the droplet speed $)$ takes values of the order of $10^{-2}$ in our experiments, which indicates that the surface tension is dominant, and consequently the droplet deformation is negligible. To measure the diameter, the droplet edge was detected in the image with a subpixel resolution method ${ }^{25}$, and the resulting contour was fitted by a circle (Fig. 3). In some cases, the edge detection method failed, and the contour was manually determined at the pixel level. We analyzed $n=10$ images for each experimental realization. In this way, we obtained a set of values $\left\{d_{i}\right\}(i=1,2, \ldots, n)$ for the Ith experimental realization. The outcome of that realization is the mean value $d_{I}=n^{-1} \sum_{i=1}^{i=n} d_{i}$. The final result is the value averaged over $N=10$ experimental realizations, $d=N^{-1} \sum_{I=1}^{I=N} d_{I}$, and the error is the corresponding standard deviation. We checked that the diameter did not significantly decrease due to evaporation along the droplet path between the upper and lower plates.

The charge of the first-emitted drop is that produced by the 


\begin{tabular}{cccccccccc}
\hline Liquid & $\rho\left(\mathrm{kg} / \mathrm{m}^{3}\right)$ & $\mu(\mathrm{mPa} \mathrm{s})$ & $\gamma(\mathrm{mN} / \mathrm{m})$ & $K(\mu \mathrm{S} / \mathrm{m})$ & $\varepsilon$ & $V_{0 \min }(\mathrm{kV})$ & $V_{0 \max }(\mathrm{kV})$ & $\mathscr{V}_{\min }(\mu \mathrm{l})$ & $\mathscr{V} \max (\mu \mathrm{l})$ \\
\hline 1-Propanol & 803 & 1.94 & 23.7 & 21 & 20.3 & - & - & - & - \\
1-Octanol & 827 & 7.20 & 23.5 & 2.6 & 10 & 2.55 & 3.75 & 8.0 & 7.0 \\
n-Decanol & 828 & 11.8 & 28.0 & 0.94 & 7.6 & 2.78 & 3.75 & 8.0 & 6.0 \\
\hline
\end{tabular}

TABLE I. Properties of the working liquids, the values of $V_{0}$ considered in our experiments, and the corresponding pendant droplet volume $\mathscr{V}$.

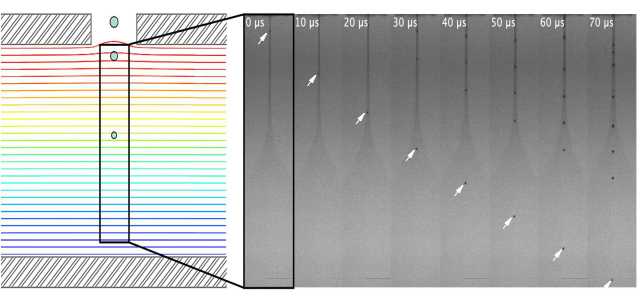

FIG. 4. Images of the first-emitted octanol droplet moving between the middle and lower plates for $V_{0}=V_{0 \mathrm{~min}}$. The arrows indicate the position of the first droplet. The darker grey region is a mirror image of the electrified meniscus located between the upper and middle plates. The left-hand image shows the isolines of voltage calculated with COMSOL.

ions trapped in that drop during the ejection process. Pure-ion evaporation is expected to occur only for a small set of liquids, such as concentrated sulfuric acid solutions, ionic liquids, and liquid metals, which have not been used in this work. In addition, we verified that liquid evaporation did not take place over the drop path. Therefore, we can safely suppose that both the diameter and charge of the first-emitted droplet remain constant all the way down to the lower plate. To measure the droplet charge, we analyzed the path followed by the droplet in the gap between the middle and lower electrode (Fig. 4) The applied electric field in this region is practically uniform because the perturbation produced by the electrified tapering meniscus is shielded by the middle plate. In fact, and as shown in Fig. 4, the distortion produced by the middle plate orifice in the electric field is negligible in the region analyzed. Also, we verified that the electric field produced by both the droplets moving behind the first droplet and the charge induced in the lower plate by the first-emitted droplet was negligible as compared to the applied electric field. The distance between the first and second droplet in Fig. 4 slightly increases over time, which indicates that the charge density of the former is significantly greater than that of the latter. For $V_{0}=V_{0 \max }$, the octanol droplet size was so small that the drag force made the subsequently emitted droplets catch the first. For this reason, we do not report the value for the droplet charge in that case.

The droplets in the images used to determine the charge corresponded to spots 1-2 pixels in diameter for octanol, and 4-6 pixels for decanol, which explains why the diameter had to be measured from a different set of experiments. The centers of those spots were determined manually. The droplet charge was obtained by fitting the path calculated from the momen- tum equation to the experimental one. The droplet path $y(t)$ obeys to the differential equation

$$
\rho \frac{\pi d^{3}}{6} \frac{d^{2} y}{d t^{2}}=q E-\frac{1}{2} \rho_{a} \frac{\pi d^{2}}{4} C_{D}\left(\frac{d y}{d t}\right)^{2} .
$$

In the above expression, $E$ is the electric field between the middle and lower plates,

$$
C_{D}(\mathrm{Re})=\frac{24}{\operatorname{Re}} f(\mathrm{Re})
$$

is the drag coefficient,

$$
f(\operatorname{Re})=10^{0.261 \mathrm{Re}^{0.369}-0.105 \mathrm{Re}^{0.431}-0.124 /\left[1+\log \left(\operatorname{Re}^{2}\right)\right]}
$$

is the correction due to the finite value of the Reynolds number $\operatorname{Re}=\rho_{a}|d y / d t| d / \mu_{a}$, and $\mu_{a}$ is the air viscosity ${ }^{26}$. We have neglected the effect of gravity and the contribution of the droplet virtual mass to the drag force due to the droplet acceleration ${ }^{27}$. It is worth mentioning that the contribution of the acceleration term on the left-hand side of Eq. (1) is smaller than that of the other terms but it cannot be neglected to get an accurate result. As mentioned in Sec. II A, the distances between electrodes as well as the electrical resistances of the two resistors (Fig. 2) were chosen so that the magnitude of the electric field between the upper and middle electrodes is similar to that obtained between the middle and lower one. In this way, the first-emitted droplet does not experience a large acceleration after crossing the middle plate, the second-order derivative on the left-hand side of Eq. (1) plays a secondary role, and, therefore, time discretization errors become smaller. The Reynolds number takes values in the range 5-10. For this interval of Re, the deviation from the Stokes law given by $f(\mathrm{Re})$ [Eq. (2)] is significant too. We have verified that the results are practically the same when $f(\mathrm{Re})$ is calculated with other commonly used approximations ${ }^{28}$

As mentioned above, it is not possible to measure accurately the droplet diameter in the same experimental realization as that used to determine the charge. For this reason, $d$ in Eq. (1) is an input variable whose value is obtained from the experiments conducted to determine it, as described above. This is probably the major limitation of the experimental procedure proposed in this work. To reduce the influence of the diameter $d$ on the calculation of the charge $q$, we regard as optimization variable $q / q_{R}$ instead of $q$. In this way, Eq. (1) becomes

$$
\frac{\rho d^{3 / 2}}{12\left(2 \varepsilon_{o} \gamma\right)^{1 / 2}} \frac{d^{2} y}{d t^{2}}=\frac{q}{q_{R}} E-\frac{\rho_{a} d^{1 / 2}}{16\left(2 \varepsilon_{o} \gamma\right)^{1 / 2}} C_{D}\left(\frac{d y}{d t}\right)^{2} .
$$




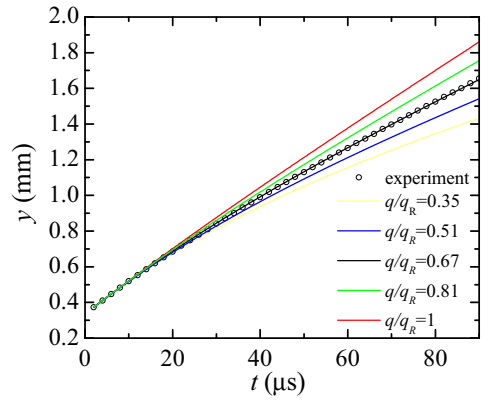

FIG. 5. Experimental path $y(t)$ of the first octanol droplet for $V_{0}=$ $V_{0 \mathrm{~min}}$ and positive polarity (symbols), and the solution to Eq. (4) calculated for $d=6.0 \mu \mathrm{m}, v_{0}=18.5 \mathrm{~m} / \mathrm{s}$, and different values of $q / q_{R}$ as indicated by the labels.

Since $C_{D} \sim d^{-0.735}$ for the interval 3-7 of Re in our experiments, $d^{1 / 2} C_{D} \sim d^{-0.235}$, the drag force in Eq. (4) is little sensitive to the value of $d$, and so is $q / q_{R}$.

We fitted the numerical solution of Eq. (4) to the experimental droplet path using $q / q_{R}$ and the initial velocity $v_{0}$ as the optimization variables. In the fitting, the diameters are randomly sampled from the normal distribution whose mean value and standard deviation are those calculated in the experiments described above. For each diameter, we calculated $q / q_{R}$ and $v_{0}$ leading to the minimum mean square deviation between the numerical and experimental paths. In this way, we obtained a set of $n$ pair of values $\left\{\left(q / q_{R}\right)_{i}, v_{0 i}\right\}(i=1,2, \ldots, n)$ for the Ith experimental realization. The outcome of that realization are the mean values $\left(q / q_{R}\right)_{I}=n^{-1} \sum_{i=1}^{i=n}\left(q / q_{R}\right)$ and $v_{0 I}=n^{-1} \sum_{i=1}^{i=n} v_{0 i}$. As expected, the sets of values $\left\{\left(q / q_{R}\right)_{i}, v_{0 i}\right\}$ exhibit very small standard deviations, and both $\left(q / q_{R}\right)_{I}$ and $v_{0 I}$ practically coincide with the values obtained by considering the mean diameter in the fitting.

Figure 5 shows the experimental path and the solution of Eq. (4) calculated for different values of $q / q_{R}$ and fixed values of $d$ and $v_{0}$. As can be observed, there is a remarkable agreement for $q / q_{R}=0.67$, while the rest of the values of $q / q_{R}$ lead to significant deviations from the experimental data. It is worth noting that the paths in Fig. 5 significantly deviate from each other for $t \gtrsim 50 \mu \mathrm{s}$. Therefore, the fitting effectively uses hardly half the experimental path to determine the droplet charge. This means that, if the distance between the middle and lower plates were reduced, e.g., by half, the technique sensitivity would not be enough to measure the effects detected in this work.

The final results of the experiments for measuring the droplet charge and velocity are the values averaged over $N=5$ experimental realizations, $q / q_{R}=N^{-1} \sum_{I=1}^{I=N}\left(q / q_{R}\right)_{I}$ and $v_{0}=$ $N^{-1} \sum_{I=1}^{I=N} v_{0 I}$, and the errors are the corresponding standard deviations. The electric charge $q$ is obtained as $q=\left(q / q_{R}\right) q_{R}$, where $q_{R}$ is calculated from the mean droplet diameter. The error is obtained from those of $\left(q / q_{R}\right)$ and $q_{R}$ with the stan-

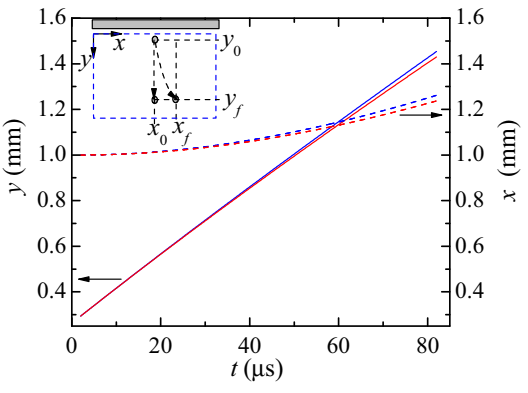

FIG. 6. Comparison between the paths $y(t)$ and $x(t)$ followed by a decanol droplet when the electric field is applied vertically (solid lines) and horizontally (dashed lines), respectively. The red and blue lines correspond to the paths calculated for $0.95 q$ and $1.05 q$, respectively ( $q$ is the electric charge measured in one of the experments). The blue square in the sketch represents the frame of the images acquired in the experiments.

dard formula of propagation of uncertainty ${ }^{29}$.

We devote the rest of this section to examine two alternatives to the proposed experimental method which could lead to more accurate results. A natural question is whether the sensitivity of the experimental technique for measuring the droplet charge increases when the electric field is applied horizontally. Figure 6 compares the paths followed by a decanol droplet when the electric field is applied vertically (solid lines) and horizontally (dashed lines). The red and blue lines correspond to the paths calculated for $0.95 q$ and $1.05 q$, respectively. The results were obtained by integrating the corresponding differential equations simulating the conditions of one of the experiments. The difference between the final positions is around 24 and $25 \mu \mathrm{m}$ when the electric field is applied vertically and horizontally, respectively. These values correspond to around 12 pixels in the experimental images. Therefore, the two configurations exhibit practically the same sensitivity, and that sensitivity allows us to measure the electric charge with errors below $10 \%$. Nevertheless, the setup for the axial field is much more convenient because it does not interfere with the image acquisition system.

As mentioned above, the major limitation of our experimental technique is probably our inability to measure the droplet diameter and charge in the same experimental realization. In his founding experiment to determine the elementary charge, Millikan ${ }^{30}$ solved this problem by measuring the downward terminal speed reached by an oil droplet in the absence of the electrical force, and the upward terminal speed when the droplet motion was inverted by the action of a known electric field. Measuring the stopping potential ${ }^{31}$ might in principle provide accurate values of the droplet charge as well. However, and given the large speed $(\sim 20 \mathrm{~m} / \mathrm{s})$ at which the droplet is ejected, these procedures would require inverting voltages of the order of kilovolts in times of the order of tens of $\mu \mathrm{s}$, which considerably complicates the experimental pro- 


\begin{tabular}{cccccccc}
\hline Liquid & $\varepsilon$ & $\delta_{\mu}$ & $B_{e \min }$ & $B_{e \max }$ & $\mathscr{V}_{\min }^{*}$ & $\mathscr{V}_{\max }^{*}$ & $B$ \\
\hline 1-Propanol & 20.3 & 2.96 & - & - & - & - \\
1-Octanol & 10 & 1.61 & 2.13 & - & 1.00 & - & 1.34 \\
n-Decanol & 7.6 & 1.55 & 2.16 & 3.95 & 1.00 & 0.75 & 1.16 \\
\hline
\end{tabular}

TABLE II. Values of the dimensionless parameters characterizing the experiments

cedure.

\section{RESULTS AND DISCUSSION}

Consider a pendant drop of volume $\mathscr{V}$ and triple contact line radius $R_{0}$. For a fixed geometry, an externally applied voltage $V_{0}$ produces electrohydrodynamic tip streaming within a small hypervolume of the parameter space defined by the dimensionless numbers $\left\{\delta_{\mu}, \varepsilon, B_{e}, \mathscr{V}^{*}, B\right\}$, where $B_{e}=\varepsilon_{0} V_{0}^{2} /\left(R_{0} \gamma\right)$ is the electric Bond number, $\mathscr{V}^{*}=\mathscr{V} / R_{0}^{3}$ is the dimensionless volume, and $B=\rho g R_{0}^{2} / \gamma$ is the gravitational Bond number, and $g$ is the gravitational acceleration. The first two parameters involve only physical properties of the liquid and outer medium, as explained in the Introduction, while the last three parameters account for the geometrical and electrical boundary conditions. In principle, the polarity of the voltage also intervenes in the problem. In this section, we present experimental results obtained for different values of the dimensionless parameters mentioned above. Table II shows only the values for which the diameter of the first emitted droplet could be measured. This requisite considerably reduces the parameter space hypervolume mentioned above. The pendant droplet volume $\mathscr{V}^{*}$ was slightly adjusted to produce the tip streaming ejection between the two electrodes.

Table III shows the diameters $d_{+}$and $d_{-}$of the droplets of octanol and decanol ejected with positive and negative polarities, respectively. For $V_{0}=V_{0 \mathrm{~min}}$, the volume of the octanol droplet emitted with positive polarity is about $23 \%$ larger than that of the one ejected when the polarity is inverted. For $V_{0}=V_{0 \max }$, the size of the octanol droplet decreases down to around 2-3 $\mu \mathrm{m}$, which falls below the resolution limit of our experimental technique. This implies that the magnitude of the applied electric field significantly affects the ejection process in this case. In the case of decanol, this influence becomes much smaller, and the polarity has opposite effects for $V_{0}=V_{0 \min }$ and $V_{0}=V_{0 \max }$. The influence of the electric field magnitude on the measurements indicates that, if the voltage drop between the upper and middle plates were kept constant, and the distance between them were varied, then the measurements would change significantly for octanol, and to some little extent for decanol.

Table IV shows the charges $q_{+}$and $q_{-}$of the droplets of octanol and decanol ejected with positive and negative polarities, respectively. The values of $q / q_{R}$ obtained for octanol are significantly larger than those measured for decanol. For octanol, $\left(q / q_{R}\right)_{+}>\left(q / q_{R}\right)_{-}$, while this ratio is hardly affected by polarity in the decanol case. As shown in Table V, drople velocities one order of magnitude larger than $v_{o}$ were obtained

\begin{tabular}{|c|c|c|c|c|}
\hline & \multicolumn{2}{|c|}{$V_{0}=V_{0 \min }$} & \multicolumn{2}{c|}{$V_{0}=V_{0 \max }$} \\
\hline & $d_{+}(\mu \mathrm{m})$ & $d_{-}(\mu \mathrm{m})$ & $d_{+}(\mu \mathrm{m})$ & $d_{-}(\mu \mathrm{m})$ \\
\hline Octanol & $6.0 \pm 0.5$ & $5.6 \pm 0.6$ & - & - \\
\hline Decanol & $9.9 \pm 0.4$ & $8.9 \pm 0.7$ & $9.4 \pm 0.8$ & $9.9 \pm 0.5$ \\
\hline
\end{tabular}

TABLE III. Diameters $d_{+}$and $d_{-}$of the first droplet emitted with positive and negative polarities, respectively.

in all the cases.

The results obtained in this work are compared with previous experimental and numerical results for the droplet diameter ${ }^{14}$, as well as with previous simulation results for the electric charge ${ }^{14,17}$. Our measurements of the droplet diameter are consistent with the exponent of the scaling law derived by Gañán-Calvo et al. ${ }^{14}$. However, the prefactor of that scaling law leads to an overestimation of the droplet diameter. It must be noted that this scaling law does not account for the influence of the voltage magnitude and polarity, which play a non-negligible role in our experiments. The predictions of both Collins et al. ${ }^{17}$ and Gañán-Calvo et al. ${ }^{14}$ slightly underestimates the ratio $q / q_{R}$ measured in our experiments. As mentioned in the Introduction, during the ultra-fast ejection of the first-emitted droplet, the mother drop apex suddenly stretches, giving rise to a large fresh free surface both per unit volume and time. In fact, this process takes place with a characteristic time comparable to the electric relaxation time. For this reason, conduction of charge from the bulk to the newly created surface cannot be described in terms of the constant (hydrostatic) conductivity considered in those theoretical predictions. When the polarity is inverted, the chemical composition of the ions transferred to the free surface changes, and so does the mobilities of those ions. This effect is not accounted for by the ohmic model either, in which the conductivity is assumed independent of the polarity. The polarity effect is expected to become more noticeable for liquids with anions and cations of very different sizes (mobilities), such as ionic liquids. Unfortunately, we did not succeed in extending our analysis to those liquids. These deviations from the ohmic approximation are expected to leave their mark on the diameter and charge of the first-emitted droplet.

Overall, our results show the complexity of the present electrohydrodynamic phenomenon, revealing the role played by factors commonly neglected in the first-order approximation. Models such as that solved by Pillai et al. ${ }^{18}$ are potentially capable of accounting for the electrokinetic effects mentioned above if the parameters characterizing the present electrohydrodynamic phenomenon are appropriately selected. However, their results do not consider polarity effects. In fact, Pillai et al. ${ }^{18}$ use "symmetric anion and cations" with equal diffusivities (or mobilities), which is a convenient simplification but not applicable to our case. Appropriate characterization of the present electrochemical process and the further resolution of the complete model, constitutes a formidable task given the intricacy of the problem.

Unfortunately, we could not extend our analysis to other liquids to explore the dependency of $d / d_{o}$ and $q / q_{R}$ on their corresponding scaled variables $\zeta_{d}$ and $\zeta_{R}$. This probably requires increasing both the electrode-to-electrode distance and 


\begin{tabular}{|c|c|c|c|c|}
\hline & \multicolumn{2}{|c|}{$V_{0}=V_{0 \min }$} & \multicolumn{2}{c|}{$V_{0}=V_{0 \max }$} \\
\hline & $q_{+}(\mathrm{fC})$ & $q_{-}(\mathrm{fC})$ & $q_{+}(\mathrm{fC})$ & $q_{-}(\mathrm{fC})$ \\
\hline Octanol & $40 \pm 8$ & $32 \pm 6$ & - & - \\
\hline Decanol & $72 \pm 7$ & $61 \pm 10$ & $67 \pm 8$ & $74 \pm 7$ \\
\hline
\end{tabular}

\begin{tabular}{|c|c|c|c|c|}
\hline & \multicolumn{2}{|c|}{$V_{0}=V_{0 \min }$} & \multicolumn{2}{c|}{$V_{0}=V_{0 \max }$} \\
\hline & $\left(q / q_{R}\right)_{+}$ & $\left(q / q_{R}\right)_{-}$ & $\left(q / q_{R}\right)_{+}$ & $\left(q / q_{R}\right)_{-}$ \\
\hline Octanol & $0.66 \pm 0.09$ & $0.59 \pm 0.07$ & - & - \\
\hline Decanol & $0.52 \pm 0.04$ & $0.51 \pm 0.06$ & $0.53 \pm 0.01$ & $0.52 \pm 0.03$ \\
\hline
\end{tabular}

TABLE IV. Electric charge $q_{+}$and $q_{-}$(left) and electric charge in terms of the Rayleigh charge, $\left(q / q_{R}\right)_{+}$and $\left(q / q_{R}\right)_{-}$(right), of the first droplet emitted with positive and negative polarities, respectively.

\begin{tabular}{|c|c|c|c|c|}
\hline & \multicolumn{2}{|c|}{$V_{0}=V_{0 \min }$} & \multicolumn{2}{c|}{$V_{0}=V_{0 \max }$} \\
\hline & $v_{0+}(\mathrm{m} / \mathrm{s})$ & $v_{0-}(\mathrm{m} / \mathrm{s})$ & $v_{0+}(\mathrm{m} / \mathrm{s})$ & $v_{0-}(\mathrm{m} / \mathrm{s})$ \\
\hline Octanol & $18.2 \pm 0.7$ & $17 \pm 1$ & - & - \\
\hline Decanol & $15.5 \pm 0.3$ & $16.1 \pm 0.6$ & $18.3 \pm 0.6$ & $17.8 \pm 0.5$ \\
\hline
\end{tabular}

\begin{tabular}{|c|c|c|c|c|}
\hline & \multicolumn{2}{|c|}{$V_{0}=V_{0 \min }$} & \multicolumn{2}{c|}{$V_{0}=V_{0 \max }$} \\
\hline & $\left(v_{0} / v_{o}\right)_{+}$ & $\left(v_{0} / v_{o}\right)_{-}$ & $\left(v_{0} / v_{o}\right)_{+}$ & $\left(v_{0} / v_{o}\right)_{-}$ \\
\hline Octanol & $8.9 \pm 0.4$ & $8.3 \pm 0.5$ & - & - \\
\hline Decanol & $10.1 \pm 0.2$ & $10.5 \pm 0.4$ & $12.0 \pm 0.4$ & $11.6 \pm 0.3$ \\
\hline
\end{tabular}

TABLE V. Velocity $v_{0+}$ and $v_{0-}$ (left) and velocity in terms of the characteristic velocity, $\left(v_{0} / v_{o}\right)_{+}$and $\left(v_{0} / v_{o}\right)_{-}$(right), of the first droplet emitted with positive and negative polarities, respectively, measured right beyond the middle plate orifice.

the applied voltage up to values unreachable with our experimental setup. It is also necessary to select appropriately the liquid conductivity and viscosity within a very narrow range. In fact, we succeeded in measuring the droplet diameter and charge for $\delta_{\mu} \simeq 1.6$ (Table II). However, when the value of this parameter was varied, different phenomena prevented us from conducting the experiment properly. For the sake of illustration, Fig. 8 shows the electrohydrodynamic response of propanol $\left(\delta_{\mu}=2.96\right)$ for $V_{0}=3.75 \mathrm{kV}$. A stream of tiny droplets is ejected from the apex of the mother parent drop for times in the interval 0-3 $\mu \mathrm{s}$. These droplets are produced by the growth of the varicose instability. The surface charge in the liquid thread located within the observation window increases over time, which gives rise to a sequence of nonaxisymmetric instabilities. The kink, ramified, and multiplebranch instabilities, frequently observed in electrospray ex periments as the electric Bond number increases ${ }^{32}$, arise in the course of the experiment.

The electrohydrodynamic response of low-conductivity pendant drops to a step-change in the electric field magnitude has been examined both numerically and experimentally on numerous occasions. Under certain conditions, tiny droplets are emitted from the tip of the electrified droplet before the steady cone-jet mode of electrospray is established. Despite the importance of this electrohydrodynamic process at both the fundamental and technological levels, the electric charge of the first-emitted droplet has not as yet determined experimentally. In this work, we have presented an experimental technique to determine with unprecedented accuracy the diameter of that droplet and to measure its electric charge. We have discussed the advantages of our method over possible alternatives. The major limitation of our technique lies in the narrow range of electrical conductivity and viscosity within which the method can be applied.

The proposed method has been used to examine the disintegration of two alcohols, octanol and decanol, commonly used in electrospray, and whose electrical conductivities take values of the order of a few $\mu \mathrm{S} / \mathrm{m}$. The high sensitivity of our experimental technique allowed us to determine the influence of both the magnitude and polarity of the applied electric field on the size and charge of the ejected droplet. The dependency of the results on those two factors reveals the complexity of this electrohydrodynamic phenomenon. The electric charge of the first-ejected droplet lied in the interval $0.51 \lesssim q / q_{R} \lesssim 0.66$ for the two liquids analyzed. These experimental values are slightly larger than those obtained from theoretical predictions ${ }^{14,17}$. To the best of our knowledge, this the first time that the charge of the first-emitted droplet in electrospray is determined experimentally. This quantity is very important because it sets an upper bound for the charge density of the droplets emitted in the cone-jet mode of electrospray.

\section{ACKNOWLEDGMENTS}

This research has been supported by the Spanish Ministry of Economy, Industry and Competitiveness under Grants DPI2016-78887 and PID2019-108278RB, and by Junta de Extremadura under Grant GR18175.

The data that support the findings of this study are available from the corresponding author upon reasonable request.

${ }^{1}$ J. W. S. Rayleigh, "On the equilibrium of liquid conducting masses charged with electricity," Proc. Roy. Soc. 5, 110 (1881).

${ }^{2}$ G. Taylor, "Disintegration of water drops in electric field," Proc. R. Soc. Lond. A 280, 383-397 (1964)

3. M. Montanero and A. M. Gañán-Calvo, "Dripping, jetting and tip streaming," Rep. Prog. Phys. 83, 097001 (2020).

${ }^{4}$ C. G. Garton and Z. Krasucki, "Bubbles in insulating liquids: stability in an electric field," Proc. Roy. Soc. Lon. A 280, 211-226 (1964).

D. Duft, T. Achtzehn, R. Muller, B. A. Huber, and T. Leisner, "Coulomb fission: Rayleigh jets from levitated microdroplets," Nature 421, 128 (2003).

${ }^{6}$ T. A. ans R. Muller, D. Duft, and T. Leisner, "The Coulomb instability of charged microdroplets: dynamics and scaling," Eur. Phys. J. D 34, 311-313 (2005)

E. Giglio, B. Gervais, J. Rangama, B. Manil, B. A. Huber, D. Duft, R. Muller, T. Leisner, and C. Guet, "Shape deformations of surface-charged microdroplets," Phys. Rev. E 77, 036319 (2008).

${ }^{8} \mathrm{Q}$. Brosseau and P. M. Vlahovska, "Streaming from the equator of a drop in an external electric field," Phys. Rev. Lett. 119, 034501 (2017).

${ }^{9}$ L. Oddershede and S. R. Nagel, "Singularity during the onset of an electrohydrodynamic spout," Phys. Rev. Lett. 85, 1234-1237 (2000).

${ }^{10}$ J. Beroz, A. J. Hart, and J. M. Bush, "Stability limit of electrified droplets," Phys. Rev. Lett. 122, 244501 (2019). 

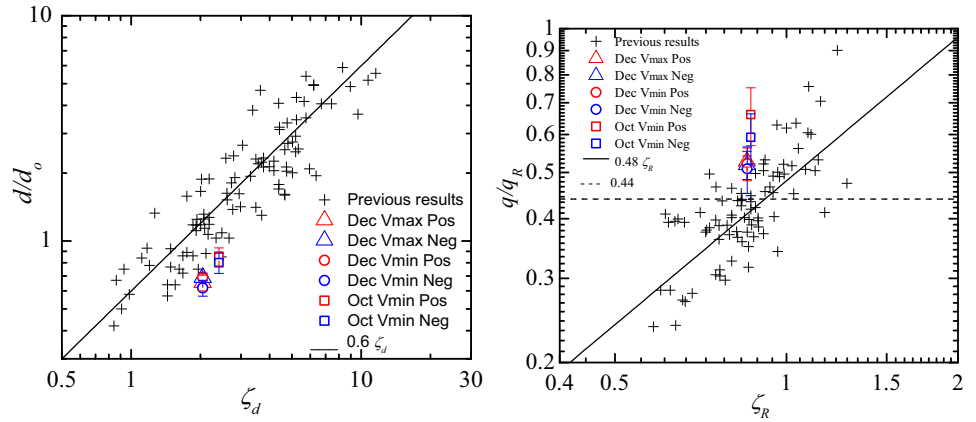

FIG. 7. $d / d_{o}\left(\zeta_{d}\right)$ (left) and $q / q_{R}\left(\zeta_{R}\right)$ (right). The crosses in the left-hand graph correspond to previous numerical and experimental results for $d / d_{0}{ }^{14}$. The crosses in the right-hand graph are previous numerical results for $q_{R} / q_{0}{ }^{14}$. The solid lines are the scaling laws $d / d_{o}=0.6 \zeta_{d}$ and $q / q_{R}=0.48 \zeta_{R}{ }^{14}$. The dashed line in the right-hand graph corresponds to the prediction $q / q_{R}=0.44^{17}$.

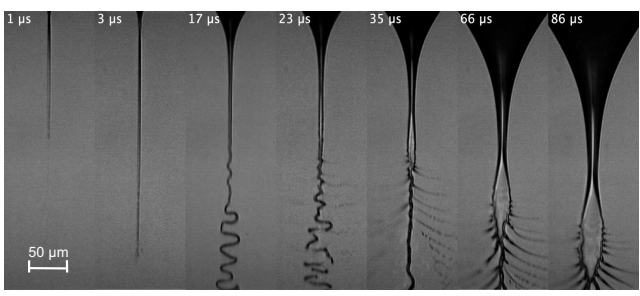

FIG. 8. Sequence of images of the electrohydrodynamic response of a propanol pendant drop following voltage switching for $V_{0}=3.75$ $\mathrm{kV}$. The instant $t=0$ approximately corresponds to the formation of the first (practically invisible) emitted droplet. The varicose, kink, ramified, and multiple-branch modes ${ }^{32}$ grow in the course of the experiment.

${ }^{11}$ U. Stachewicz, J. F. Dijksman, C. U. Yurteri, and J. C. Marijnissen, "Experiments on single event electrospraying," Appl. Phys. Lett. 91, 254109 (2007).

${ }^{12} \mathrm{M}$. D. Paine, "Transient electrospray behaviour following high voltage switching," Microfluid Nanofluid 6, 775-783 (2009).

${ }^{13}$ C. Ferrera, J. M. López-Herrera, M. A. Herrada, J. M. Montanero, and A. J. Acero, "Dynamical behavior of electrified pendant drops," Phys. Fluids $\mathbf{2 5}$ 012104 (2013)

${ }^{14}$ A. M. Gañán-Calvo, J. M. López-Herrera, N. Rebollo-Muñoz, and J. M. Montanero, "The onset of electrospray: the universal scaling laws of the first ejection," Sci. Rep. 6, 32357 (2016)

${ }^{15}$ M. A. Fontelos, U. Kindelán, and O. Vantzos, "Evolution of neutral and charged droplets in an electric field," Phys. Fluids 20, 092110 (2008)

${ }^{16}$ R. T. Collins, J. J. Jones, M. T. Harris, and O. A. Basaran, "Electrohydrodynamic tip streaming and emission of charged drops from liquid cones," Nat. Phys. 4, 149-154 (2008).

${ }^{17}$ R. T. Collins, K. Sambath, M. T. Harris, and O. A. Basaran, "Universal scaling laws for the disintegration of electrified drops," Proc. Nat. Acad. Sci. 110, 4905-4910 (2013).

${ }^{18}$ R. Pillai, J. D. Berry, D. J. E. Harvie, and M. R. Davidson, "Electrokinetics of isolated electrified drops," Soft Matter 12, 3310-3325 (2016).

${ }^{19}$ H. Dastourani, M. R. Jahannama, and A. Eslami-Majd, "A physical insight into electrospray process in cone-jet mode: Role of operating parameters," Int. J. Heat Fluid Flow 70, 315-335 (2018).

${ }^{20} \mathrm{~K}$. Mohammadi, M. R. Movahhedy, and S. Khodaygan, "A multiphysics model for analysis of droplet formation in electrohydrodynamic 3D printing process," J. Aerosol Sci. 135, 72-85 (2019).

${ }^{21} \mathrm{~N}$. Gawande, Y. S. Mayya, and R. Thaokar, "Jet and progeny formation in the Rayleigh breakup of a charged viscous drop," J. Fluid Mech. 884, A31 (2020).

${ }^{22}$ A. M. Gañán-Calvo, N. Rebollo-Muñoz, and J. M. Montanero, "Physical symmetries and scaling laws for the minimum or natural rate of flow and droplet size ejected by Taylor cone-jets," New J. Phys. 15, 033035 (2013).

${ }^{23} \mathrm{~A}$. M. Gañán-Calvo, "The surface charge in electrospraying: Its nature and its universal scaling laws," J. Aerosol Sci. 30, 863-872 (1999).

${ }^{24}$ M. G. Cabezas, A. Bateni, J. M. Montanero, and A. W. Neumann, "A new method of image processing in the analysis of axisymmetric drop shapes," Colloids Surf. A 255, 193-200 (2005).

${ }^{25} \mathrm{E}$. J. Vega, J. M. Montanero, and C. Ferrera, "Exploring the precision of backlight optical imaging in microfluidics close to the diffraction limit," Measurement 44, 1300-1311 (2011).

${ }^{26}$ R. L. C. Lemmer and C. L. Banks, "On the drag coefficient of a sphere," Powder Technol. 48, 217-221 (1986).

${ }^{27} \mathrm{G}$. K. Batchelor, An introduction to fluid dynamics (Cambridge University Press, Cambridge, Great Britain, 1994).

${ }^{28}$ Y. Hongli, F. Minqiang, L. Airong, and D. Lianping, "General formulas for drag coefficient and settling velocity of sphere based on theoretical law," Int. J. Min. Sci. Technol. 25, 219-223 (2015).

${ }^{29} \mathrm{H}$. H. Ku, "Notes on the use of propagation of error formulas," J. Res. Nat. Bur. Stand. Sec. C: Eng. Inst. 70C, 263-273 (1966).

${ }^{30}$ R. A. Millikan, "On the elementary electrical charge and the Avogrado constant," Phys. Rev. 2, 109-143 (1913)

${ }^{31} \mathrm{M}$. Gamero-Castaño, "Energy dissipation in electrosprays and the geometric scaling of the transition region of cone-jets," J. Fluid Mech. 662, 493513 (2010).

${ }^{32} \mathrm{M}$. Cloupeau and B. Prunet-Foch, "Electrohydrodynamic spraying functioning modes: A critical review," J. Aerosol Sci. 25, 1021-1036 (1994). 

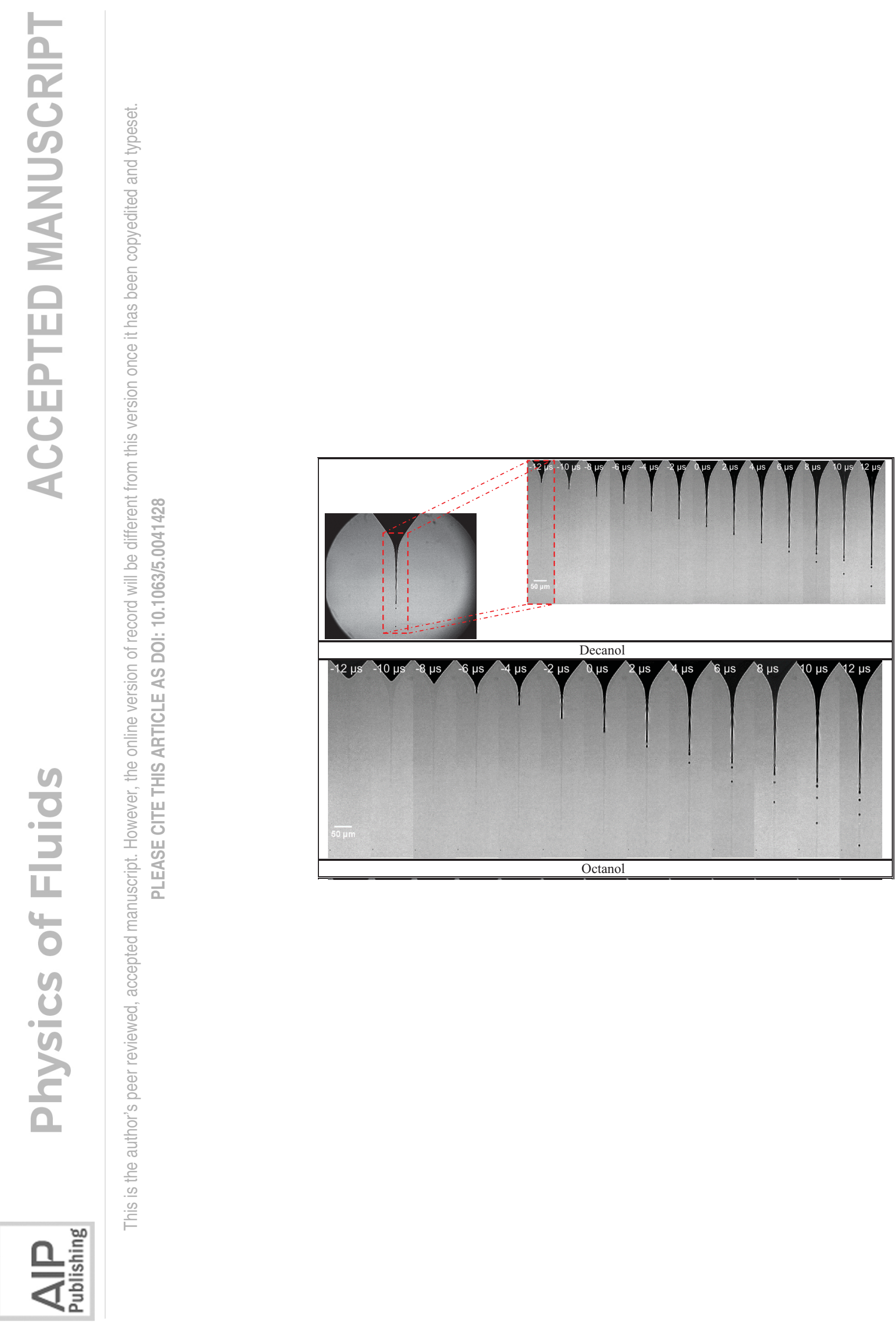


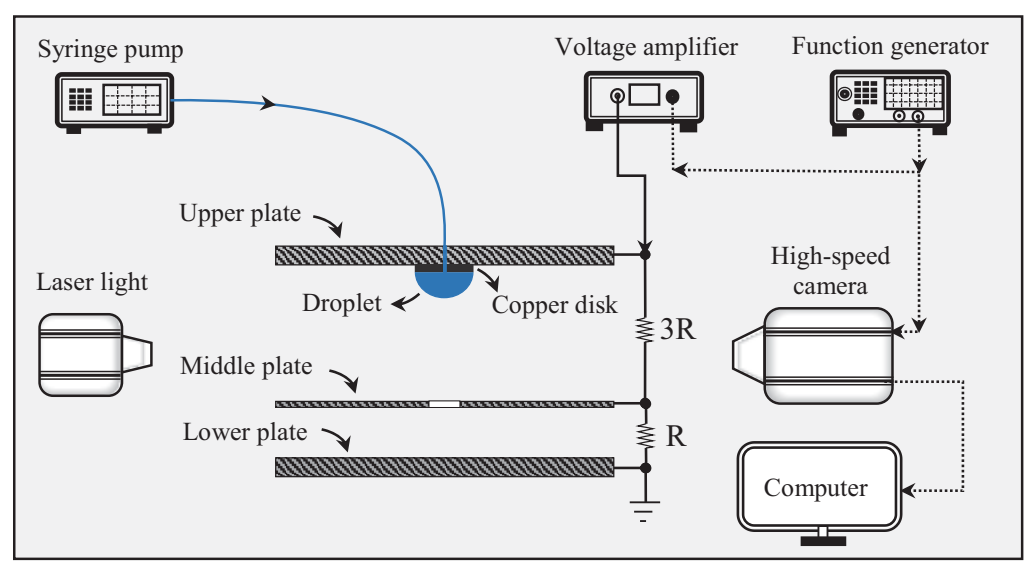




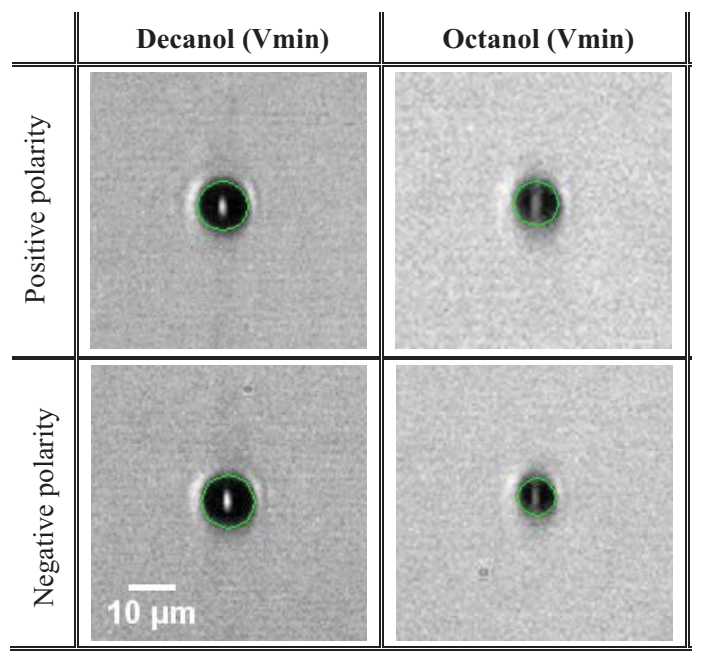

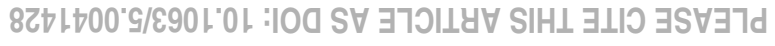

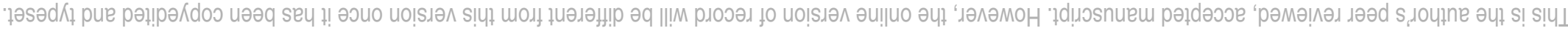



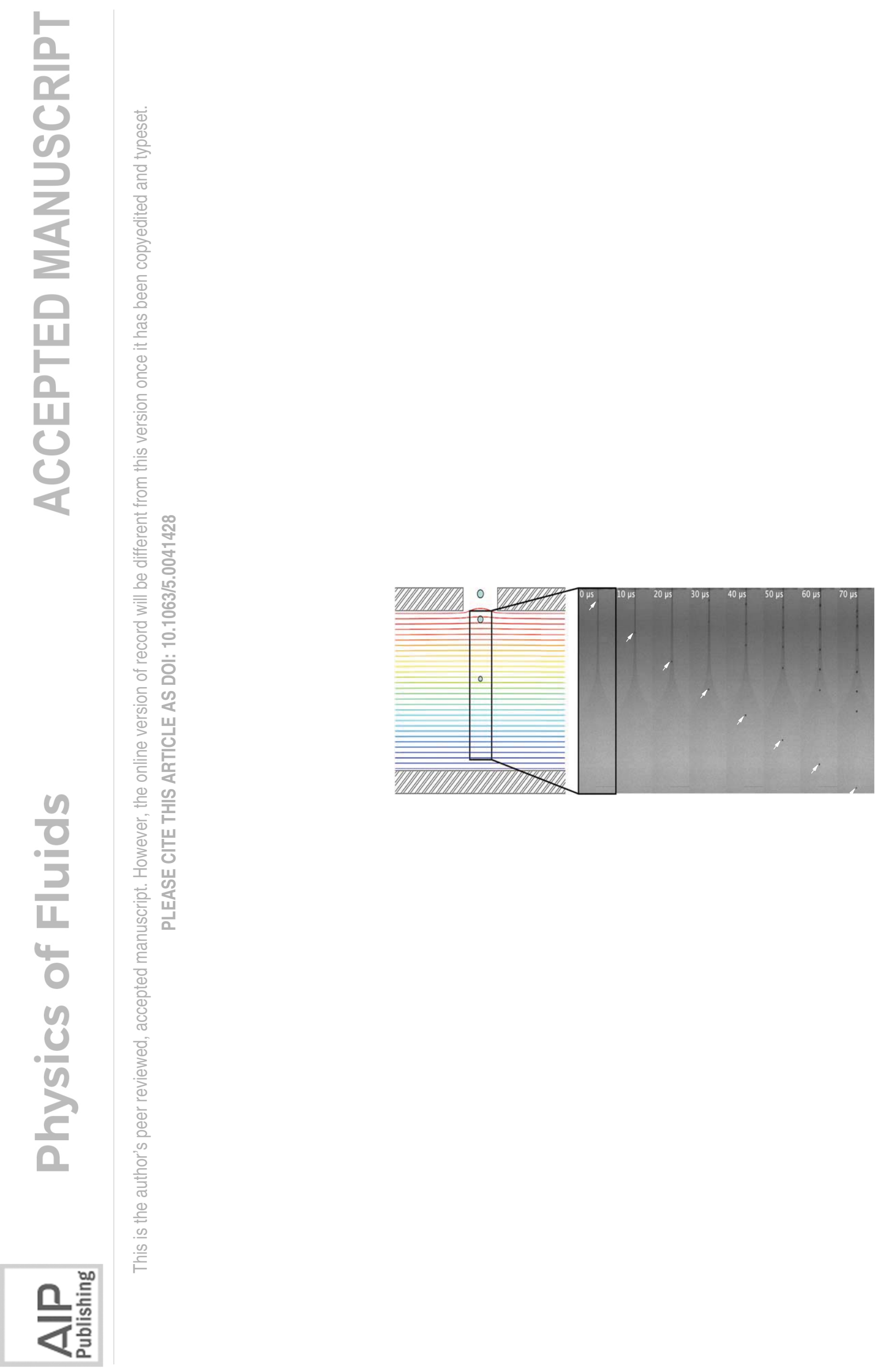

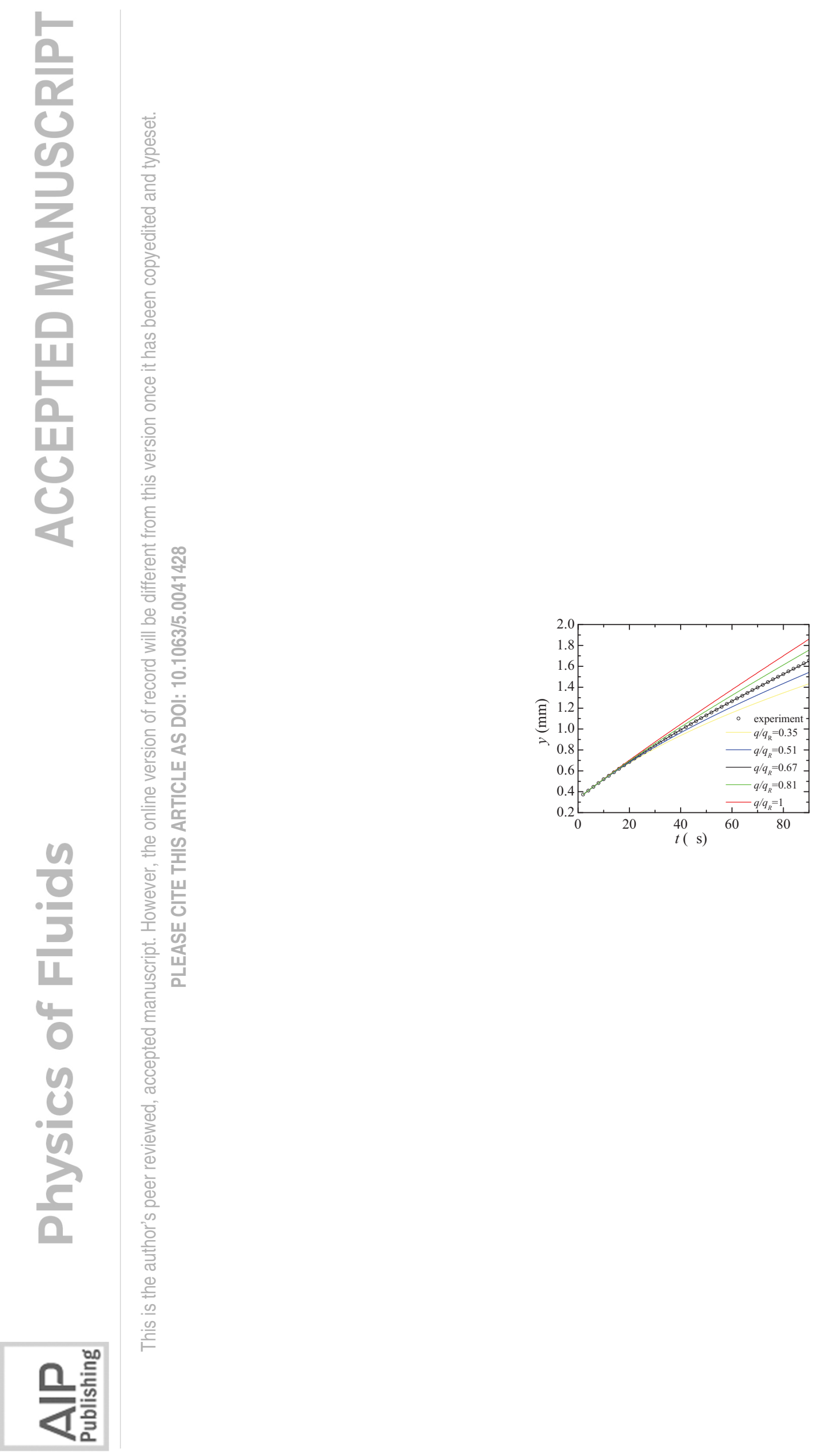

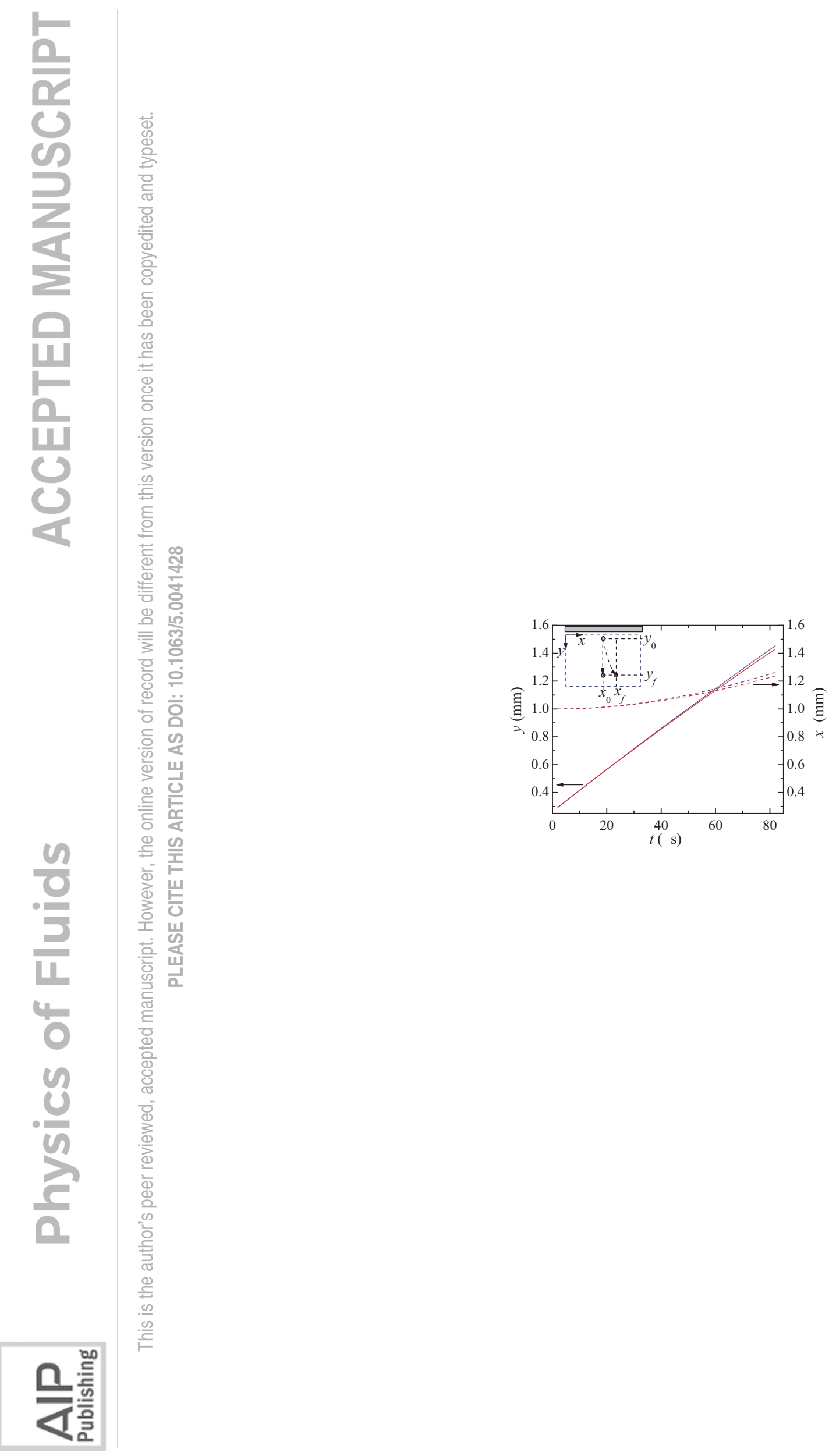

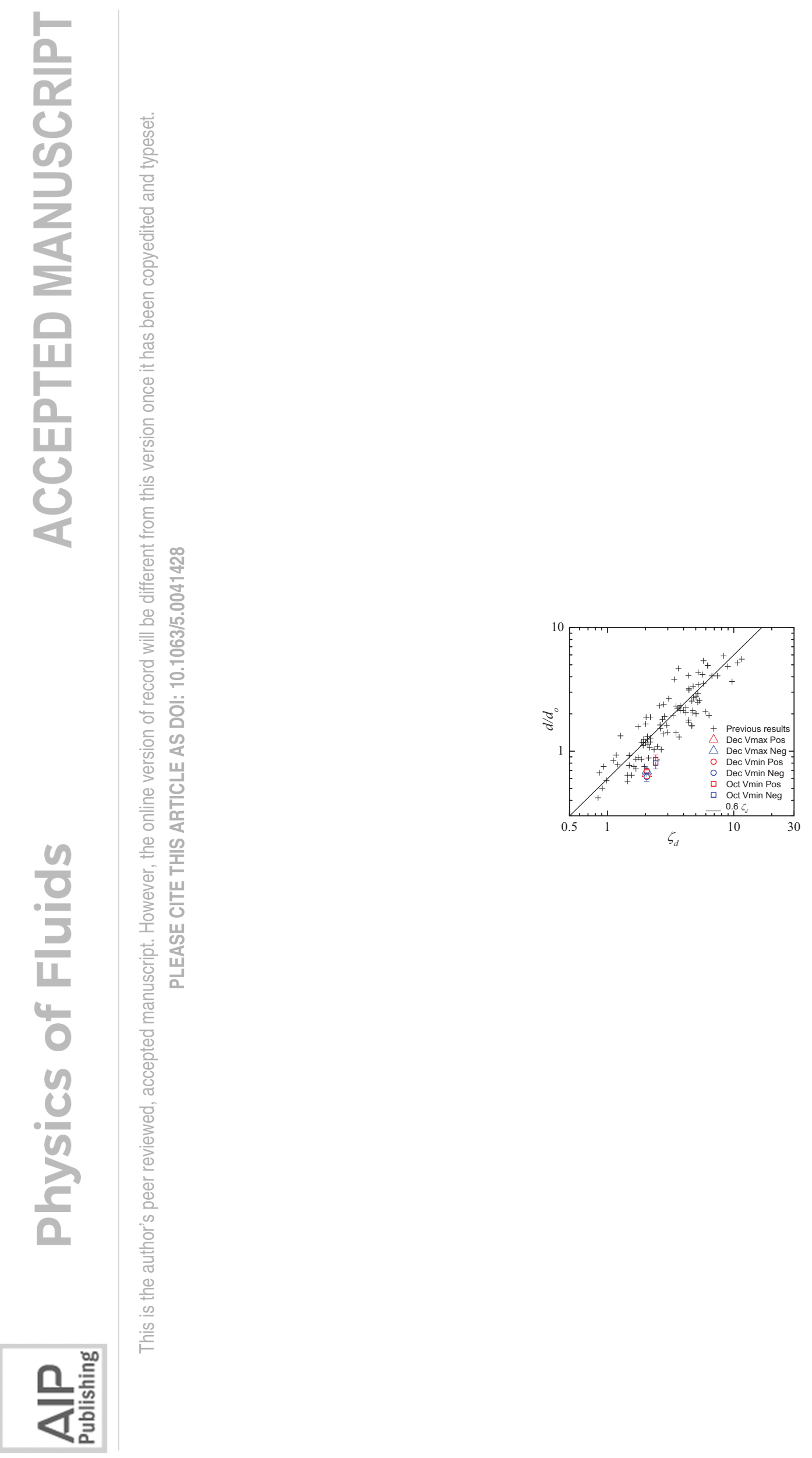

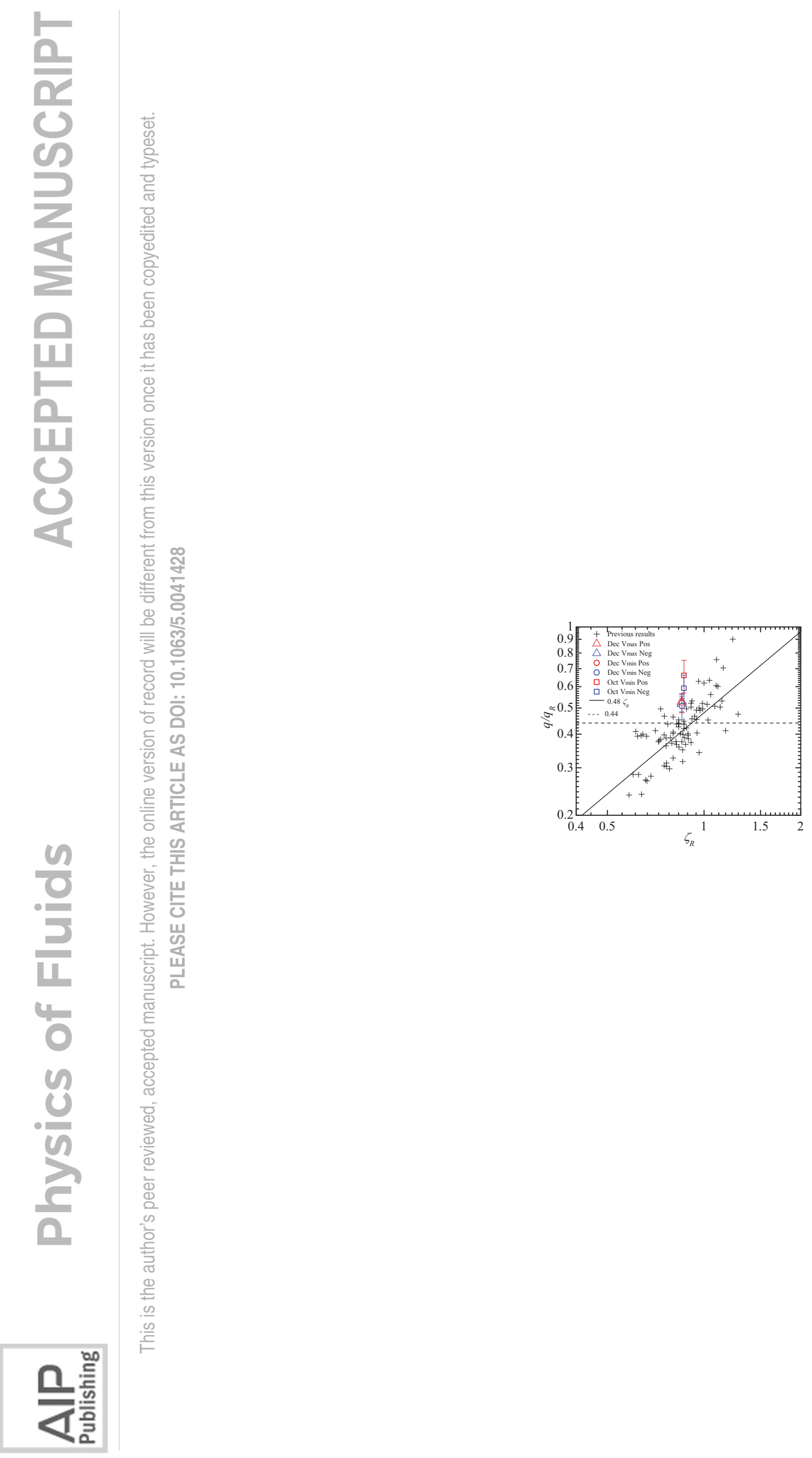


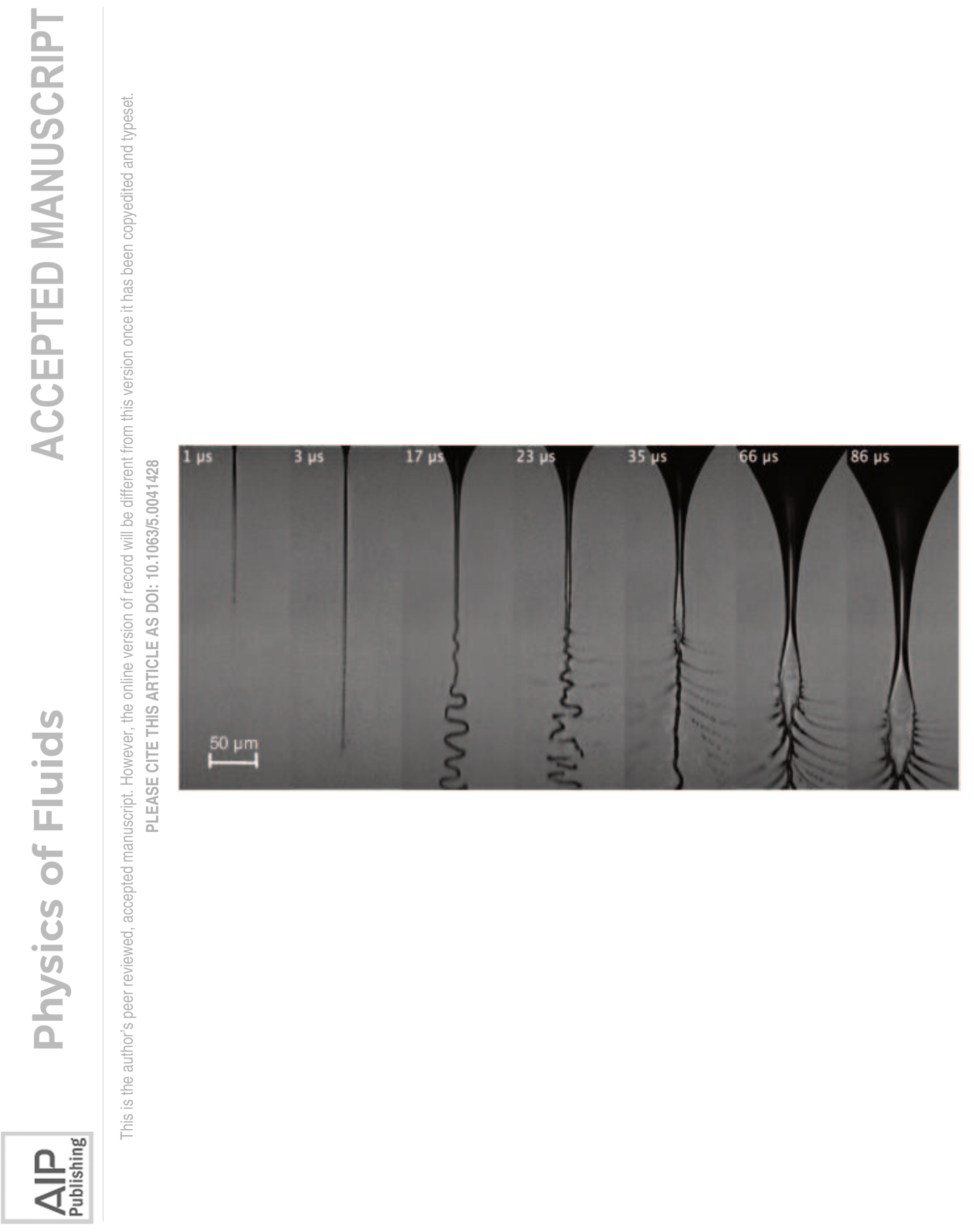

